\title{
LAS EMOCIONES POSITIVAS Y SU RELACIÓN CON EL RENDIMIENTO ACADÉMICO EN LA MATERIA DE MÉTODOS DE INVESTIGACIÓN
}

\section{POSITIVE EMOTIONS AND THEIR RELATIONSHIP WITH ACADEMIC PERFORMANCE IN THE FIELD OF RESEARCH METHODS}

\author{
Annia E. Vives Iglesias \\ Seminario Teológico Adventista de Cuba, Cuba \\ anniaevi25@gmail.com \\ https://orcid.org/0000-0001-9057-0266 \\ Miguel A. Alemany Naveiras \\ Universidad de Montemorelos, México \\ malemany@um.edu.mx \\ https://orcid.org/0000-0002-0683-8803 \\ Elvis Hernández Rodríguez \\ Seminario Teológico Adventista de Cuba, Cuba \\ ptrelvishr@gmail.com \\ https://orcid.org/0000-0002-3496-4313 \\ Tomás F. Cahuich Cahuich \\ Universidad de Montemorelos, México \\ tomca@um.edu.mx \\ https://orcid.org/0000-0002-3016-1433 \\ Amelia E. Hernández Vives \\ Universidad de Ciencias Pedagógicas Enrique José Varona, Cuba \\ ameliahedz@gmail.com \\ https://orcid.org/0000-0002-0400-5584
}

\begin{abstract}
RESUMEN
Las emociones positivas modelan la percepción de la realidad. En ambientes escolares universitarios, es necesario que exista un clima de positividad que facilite el proceso de enseñanza-aprendizaje, lo cual se puede lograr a través de actividades que generen ese tipo de emociones. Se realizó un estudio cuasi-experimental, comparativo, longitudinal y prospectivo con dos grupos de estudiantes de primer año de la licenciatura en Teología en la materia de Métodos de Investigación. En el grupo experimental se realizaron actividades planificadas que generaran emociones positivas durante los primeros cinco a siete minutos de clase, comprobando la influencia de
\end{abstract}




\section{EMOCIONES POSITIVAS Y RENDIMIENTO ACADÉMICO}

un clima de positividad sobre el desempeño en la asignatura. Se utilizó otro grupo control para comparación, donde no se hizo intervención alguna. Ambos grupos tuvieron una composición demográfica similar. Al someter los resultados a análisis estadísticos, el grupo experimental obtuvo mejores calificaciones en los productos de aprendizaje y en la nota final de la materia. Se propone incentivar el uso de las herramientas que la psicología positiva ofrece en función de la educación integral, al reproducir este tipo de intervenciones generadoras de emociones positivas en contextos educativos más amplios. Los beneficios serán más abarcantes que un mejor rendimiento académico, pues el aumento del bienestar subjetivo se incrementa proporcionalmente a este tipo de actividades.

Palabras clave: emociones positivas, rendimiento académico, ambientes de aprendizaje

\section{ABSTRACT}

Positive emotions shape the perception of reality. In university school environments, there needs to be a climate of positivity that facilitates the teaching-learning process, which can be achieved through activities that generate such emotions. A quasi-experimental, comparative, longitudinal and prospective study was carried out with two groups of first-year students of the degree in Theology in the field of Research Methods. In the experimental group, planned activities were carried out that generated positive emotions during the first 5-7 minutes of class, checking the influence of a climate of positivity on the results of the subject. Another control group was used for comparison, where no intervention was made. Both groups had a similar demographic composition. By submitting the results to statistical analysis, the experimental group obtained better grades in the learning products and in the final grade for the subject. It is proposed to encourage the use of the tools that positive psychology offers in terms of comprehensive education, by reproducing this type of intervention that generates positive emotions in broader educational contexts. Dividends will be more comprehensive than better academic performance, since the increase in subjective well-being increases proportionally to this type of activity.

Keywords: positive emotions, academic performance, positive education

\section{Introducción}

Las emociones experimentadas en cada momento son los lentes que modelan la percepción de la realidad. Si los sucesos de la vida diaria se enfrentan con una dosis extra de emociones positivas generadas de antemano e intencionalmente, la resolución de los problemas será mucho más efectiva. Esto conducirá indudablemente a una satisfacción mayor con la vida a nivel subjetivo y por ende mejores resultados objetivos en las tareas a realizar. Estos son algunos de los propósitos generales que la psicología positiva propone en una amplia gama de investigaciones y áreas de estudio (MacConville y Rae, 2012; Tarragona, 2013); con lo cual otros investigadores coinciden (Hooper, 2012; Rusk y Waters, 2013; Seligman, 2011). Todo ello sin apartarse de la más rigurosa metodología científica (Bisquerra Alzina, 2009). 
Emociones positivas: conceptos, fisiología y relación con el aprendizaje

Las emociones positivas, uno de los pilares de la teoría del bienestar, es un constructo difícil de definir, pero juega un papel muy importante en el bienestar y ha sido ampliamente investigado en varios campos del saber (Adelman y Estes, 2013; Armenta et al., 2017; Csikszentmihalyi, 2014; Elizondo Moreno et al., 2018; Frazzetto, 2014; Fredrickson, 2016; Fredrickson y Joiner, 2018; Fredrickson y Losada, 2005; Ibarrola, 2014). Su relación con el bienestar subjetivo del individuo y en su funcionamiento integral ha interesado tanto a filósofos antiguos como actuales, a psicólogos, neurocientíficos como pedagogos (Bender, 2004; Immordino-Yang et al., 2009; Rolla et al., 2011).

Las emociones se consideran estados complejos del organismo, marcados por una respuesta conductual subjetiva que está mediada por la influencia tanto del medio externo como del interno del individuo. Esto lleva a que sean consideradas fenómenos multidimensionales caracterizados por elementos cognitivos, fisiológicos, conductuales y expresivos (Ibarrola, 2014). Las investigaciones actuales sostienen que las emociones son respuestas a los cambios fisiológicos percibidos en el organismo y su contenido cognitivo se genera después de una evaluación cognitiva del estímulo, fundamentada en la experiencia previa (Ortiz Alonso, 2018). Esto lleva a plantear que "la percepción de estos cambios corporales provocados por la emoción puede jugar un papel clave en la generación de emociones conscientemente sentidas" (Ortiz Alonso, 2018, p. 53). Respecto a este componente cognitivo, se plantea que el individuo realiza dos valoraciones de los diferentes estímulos o acontecimientos a los que se expone, una al percibirlos y los etiquetan como buenos o malos e incluso son capaces de evaluarlos nuevamente en tipos específicos de malos o desagradables. Estas valoraciones específicas provocan emociones específicas. Predomina actualmente la posición cognitiva atribucional de las emociones, lo cual se fundamenta en que las evaluaciones o atribuciones "son procesos mentales que median entre los resultados vitales y las reacciones emocionales" (Ibarrola, 2014, p. 149).

Tienen componentes externos, visibles, dados por las propias alteraciones biológicas que pueden ir desde alteraciones en la conducta y en los niveles hormonales hasta las expresiones faciales. El componente interno es el sentimiento, la conciencia personal de esa emoción. Todo esto se produce por la acción sinérgica de varias estructuras cerebrales. Las más relevantes incluyen el sistema límbico, donde se producen las emociones en su forma más básica, y la corteza prefrontal, la cual tiene a su cargo la toma de decisiones, memoria y atención como parte del actuar racional. Estas áreas no son excluyentes, sino que trabajan armoniosamente (Frazzetto, 2014). Además, estas áreas cerebrales no sólo están íntimamente relacionadas con las emociones, sino también con la forma en que el individuo puede adquirir y consolidar nuevos conocimientos, lo que permite concluir la dependencia de ambas funciones, la emotiva y la del aprendizaje (Elizondo Moreno et al., 2018; Ormrod, 2005).

Las emociones ayudan a las personas a mantener su adaptación al medio, mediante respuestas conductuales, y de esta manera funcionan adecuadamente. También facilitan la adaptación del individuo 
a su entorno social. Es en ese contexto social que se producen las mejores experiencias de aprendizaje. Los aspectos emocionales y cognitivos deben considerarse unidos si se pretende un desarrollo integral del estudiante (García Retana, 2012) y lograr que la motivación sea mayor y más efectivo el aprendizaje (Ortiz Alonso, 2018). La educación reta al docente a suscitar emociones que predispongan al aprendizaje (Ibarrola, 2014).

Independientemente de las limitaciones que los estudios iniciales pudieran tener, Fredrickson y Joiner (2018) proponen que las emociones positivas cotidianas, a pesar de ser transitorias, si se trabaja en su construcción y ampliación, pueden generar una "cascada de procesos psicológicos" que facilitan un impacto duradero en el bienestar subjetivo de las personas.

Se propone que incentivar y generar emociones es parte del trabajo educativo, lo cual facilita el desarrollo cognitivo y comportamental de los estudiantes (Adelman y Estes, 2013; Sheldon y Lyubomirsky, 2006). Esto reafirma que cada acción produce una reacción. Cada cambio en el clima emocional de un aula, por pequeño que sea, tendrá un impacto importante en la conducta, motivaciones y logros de los estudiantes (Shindler, 2010; Walsh et al., 2018).

Ormrod (2005) enfatiza la necesidad de generar un ambiente motivador en el aula para preparar al estudiante para el aprendizaje y alude a las emociones positivas y a cómo pueden ser utilizadas por el profesor. Hacer que las materias a impartir sean interesantes y motivadoras, que el profesor pueda generar entusiasmo entre sus alumnos y la utilización de actividades lúdicas (Tamblyn, 2003) para reafirmar la adquisición del conoci- miento, harán que las experiencias en el aula sean positivas, recordadas y aplicadas y no consideradas amenazantes. Posibilitará que tanto niños como adultos puedan desarrollar al máximo sus potencialidades, entre ellas, sus fortalezas de aprendizaje (Hooper, 2012; Tarragona, 2014). Ambientes de positividad activan la liberación de dopamina por el cerebro y esta sensación placentera fortalece la acción sináptica favoreciendo el aprendizaje. A lo anterior puede agregarse que, si se asocia una emoción positiva a un estímulo cognitivo, este condicionamiento reforzará la capacidad de retener lo aprendido (Elizondo Moreno et al., 2018; Ibarrola, 2014).

A largo plazo, las personas que tienen más emociones positivas están más satisfechas con su vida, tienen mejores relaciones de pareja y mejor trabajo, son más eficientes y productivas e incluso viven más tiempo (Cohn et al., 2009; Fredrickson et al., 2008).

Las emociones y su relación con el aprendizaje han sido estudiadas en materias como Física o Química (Dávila Acedo et al., 2016). Se encontró que predominan las emociones positivas cuando los estudiantes obtienen buenos resultados y las negativas cuando deben resolver problemas concernientes a esas materias o hacer presentaciones orales, en una muestra de educación secundaria básica. En otras materias, como las matemáticas, se han analizado los niveles de ansiedad que presentan los estudiantes hacia la asignatura (Gómez Chacón, 2000; Ormrod, 2005) y se encontró que son afectados por las creencias previas que tenga el aprendiz acerca de ella. Esta actividad emocional consciente se convierte en un poderoso regulador de control personal, mediador en las relaciones interpersonales de los estudiantes 
y constituye un elemento clave en la forma que autorregulan su aprendizaje.

\section{Rendimiento académico y clima emocional en el aula}

Existen muchas formas de comprobar el éxito del proceso de enseñanza-aprendizaje en los diferentes ámbitos educativos, pero tradicionalmente es el rendimiento académico una de las más usadas.

Aunque no debe ser la única, el rendimiento académico toma en cuenta las puntuaciones que los estudiantes obtienen en sus exámenes parciales y finales, así como en las diferentes actividades en clase y extraclases orientadas por el profesor y que tributan a la nota final acumulada de cada materia. Así es valorado cuantitativamente el aprendizaje en una materia, partiendo de los conocimientos impartidos en el aula y de la apropiación individual que el estudiante hace de ellos, además de la gestión de su autoaprendizaje.

El concepto de rendimiento académico tiene una naturaleza multifactorial y ha sido difícil llegar a un concepto unificado. De esta manera se propone como el producto o utilidad de todas las actividades escolares, ya sean educativas como informativas. Otros lo definen como la finalidad obtenida al movilizar las fuerzas físicas y psíquicas dirigidas de manera consciente al aprendizaje, hasta quienes argumentan que es la muestra del aprovechamiento que el estudiante ha hecho del proceso de enseñanza-aprendizaje (Solano Luengo, 2015).

Algunos autores perciben que el aprendizaje logrado por los estudiantes se mide a través del rendimiento académico, lo cual permite un acercamiento a la realidad educativa (Garbanzo Vargas,
2007), y puede determinar el éxito o el fracaso y la calidad de la educación impartida (Solano Luengo, 2015). También relacionan ese logro (o fracaso) con el aprovechamiento y comprensión de los contenidos impartidos en clases (Solano Luengo, 2015), mientras que algunas investigaciones hallan una correlación entre la inteligencia emocional y el rendimiento, donde los aspectos emocionales son predictores del logro académico (Sánchez-López et al., 2015).

Los estudios del rendimiento académico en la educación superior alcanzan una mayor relevancia (Garbanzo Vargas, 2007), puesto que "un buen rendimiento académico es un indicador de ajuste escolar o de adaptación positiva al contexto educativo" (Fernández-Lasarte et al., 2019, p. 40).

Un gran número de investigaciones relacionan el rendimiento académico con diferentes factores que pueden clasificarse como determinantes sociales y personales (Barahona U., 2014), derivados del alumno, del maestro o del contexto, personales y contextuales (Fernández-Lasarte et al., 2019), cognitivos, actitudinales e instrumentales (Solano Luengo, 2015), e incluso quienes lo estudian desde los factores sociales, económicos, demográficos, geográficos $\mathrm{y}$ afectivos que puedan determinarlo (Franco Cedeño et al., 2019), los cuales van a depender del diseño y objetivos de la investigación per se, así como de la población donde se realice el estudio.

Varios trabajos revisados coinciden en que un ambiente de positividad favorece el proceso de enseñanza aprendizaje y por ende los resultados del mismo evidenciados por el rendimiento académico (Hill y Stone, 1982; Saklofske et al., 2012; Solano Luengo, 2015; Westwood, 2008). 


\section{EMOCIONES POSITIVAS Y RENDIMIENTO ACADÉMICO}

Todo lo anterior refuerza la importancia de la presente investigación, que propone generar un ambiente de emociones positivas en un aula de clases para desencadenar efectos que redundarán en un mejor aprendizaje apreciado mediante el rendimiento académico de los estudiantes.

\section{Método}

Algunos artículos hacen alusión a cómo la felicidad puede ser incrementada y sostenida por cambios positivos intencionales (Sheldon y Lyubomirsky, 2006).

Un grupo de investigadores se preguntaron si las emociones positivas provocarían efectos beneficiosos en las personas y para probar su tesis intentaron evocar emociones positivas en los participantes de sus estudios y comprobar la efectividad de las mismas. Por ejemplo, antes de responder ciertos cuestionarios, los sujetos veían un video cómico o recibían chocolates o pequeños regalos. Entre sus resultados se mostró que, cuando la gente siente emociones positivas, mejora su concentración y memoria a corto plazo, las tareas verbales son más eficientes y está también más receptiva a la información nueva. Los datos mostraron además que las emociones positivas mejoran la atención visual y la creatividad verbal, y entre los estudiantes se comprobó mejores resultados en exámenes estandarizados si existe un ambiente de positividad antes de responderlos. Teniendo en cuenta esto, se propone la teoría de "ampliar y construir", donde, generando emociones positivas de manera frecuente, se espera que los estudiantes estén más abiertos a la experiencia, a crear, construir, aprender mejor, explorar el mundo que les rodea y sus posibilidades, asimilar mejor la información y mejorar sus relaciones interpersonales (Cohn et al., 2009; Tarragona, 2013, 2014).

Teniendo estos resultados por plataforma, se realizó un estudio cuasi-experimental, comparativo, longitudinal y prospectivo cuyo propósito fue potenciar, de manera intencional, mediante actividades planificadas en un aula de clases, la generación de emociones positivas y por ende el estado de bienestar subjetivo de los estudiantes, constituyendo estas actividades el proyecto de intervención. Esto permitió comprobar cómo el estado de bienestar puede influir en el proceso de aprendizaje y cómo se manifiesta al final en el rendimiento académico, mediante el promedio obtenido en la materia Métodos de Investigación.

Esta investigación concibe el rendimiento académico como el nivel de conocimientos, destrezas, habilidades y competencias que los alumnos pueden demostrar que han alcanzado en la materia de Métodos de Investigación y que se manifiesta en la suma de las puntuaciones de las actividades y exámenes orientados con ese fin con un máximo posible de 100 puntos.

\section{Participantes}

El estudio se realizó con dos grupos de estudiantes de primer año de la licenciatura en Teología, que cursan sus estudios en el Seminario Teológico Adventista de Cuba, durante los cursos lectivos 2018-2019 y 2019-2020, durante el primer semestre de sus estudios y en la materia Métodos de Investigación. En un grupo se realizó la intervención y el otro grupo fue utilizado como control.

El estudio incluyó a todos los estudiantes que cursaban esta materia por primera vez en la carrera. Todos los estudiantes de ambos grupos accedieron a participar en el proyecto. 
El grupo donde se realizó la intervención tuvo 20 estudiantes (44.4\%) y el grupo control estuvo integrado por 25 (55.6\%), para un total de 45 alumnos.

La composición demográfica de ambos grupos fue relativamente similar. La representación femenina estuvo dada por el $22,2 \%$ y la masculina por el $77,8 \%$. Un $35,6 \%$ de los participantes eran casados y un $64,4 \%$ solteros. Hubo un $51,1 \%$ con estudios de bachillerato concluidos y un $48,9 \%$ con otras carreras universitarias. Un $77,8 \%$ era de nacionalidad cubana y un $22,2 \%$ de alguna nacionalidad extranjera.

La media de edad fue de 27.8 años, con una desviación estándar de 6.88, donde el estudiante más joven tenía 19 años y el mayor 46 .

A todos los integrantes del grupo de intervención se les brindó previamente información de los propósitos de la actividad y se obtuvo su consentimiento informado.

\section{Procedimiento/instrumento}

Se diseñó una estrategia que se insertó en el plan curricular, previa coordinación con los directivos de la institución. En el grupo de intervención, durante los primeros cinco a siete minutos de cada clase (de un total de 30 horas lectivas), se realizaron actividades diseñadas con el objetivo de generar en los estudiantes emociones positivas. Se tuvieron en cuenta los factores propuestos por Tarragona (2014) que responden al acrónimo PRISMA.

Basados en lo que Tarragona sugiere para la positividad $(\mathrm{P})$, se planificaron actividades enfocadas en emociones positivas como "la alegría, la gratitud, la serenidad, el interés, la esperanza, el orgullo, la diversión, la inspiración, el asombro y el amor" (p. 79). Por ejem- plo, escribir notas (teniendo en cuenta las prácticas narrativas y colaborativas) sobre las metas a alcanzar, notas de agradecimiento a personas específicas y compartirlas con ellas, recordar y compartir momentos cuando experimentaron gran gozo o diversión. Se contaron anécdotas curiosas y otras divertidas, se proyectaron videos motivadores cortos o de sucesos curiosos de la naturaleza. De la misma manera, se planificaron actividades que potenciaran las relaciones interpersonales $(\mathrm{R})$ entre los estudiantes, y entre los estudiantes y el profesor, y el involucramiento o flow (I) en actividades significativas para ellos y para otras personas (Csikszentmihalyi, 2014). Igualmente se programaron espacios para que los estudiantes pudieran (C) compartir sus experiencias en clase, desarrollar mediante actividades concretas las fortalezas del carácter, encontrar el significado de la vida (S) y valorar las metas alcanzadas (MA). La intención fue crear un ambiente de positividad en el aula que favoreciera el proceso de enseñanza aprendizaje, la memoria, la capacidad de pensamiento crítico, de redacción y de análisis, que se evidenció en las tareas asignadas.

Independientemente de esta actividad de intervención previa, ambos grupos recibieron la misma cantidad de clases, igual diseño metodológico e iguales requerimientos de productos del aprendizaje y exámenes. El producto de aprendizaje principal de la materia fue escribir un trabajo de investigación aplicando las reglas investigativas, de redacción y de acotación de referencias aprendidas en clases, el cual tenía un valor de la mitad de la nota final (50 puntos de 100 posibles a obtener). Esta asignación, junto a otras y los exámenes programados tributaban a la nota final del curso. 


\section{EMOCIONES POSITIVAS Y RENDIMIENTO ACADÉMICO}

El rendimiento académico de los estudiantes se midió mediante la calificación final de la materia, que incluía la suma de las actividades evaluativas realizadas durante el semestre, con un máximo de 100 puntos y un mínimo para considerar aprobada la materia de 70 puntos.

Se aplicaron pruebas estadísticas descriptivas y de variabilidad a los datos recogidos.

\section{Resultados}

Se calificó el requisito de monografía, que correspondía a un $50 \%$ de la calificación final, en una escala de 0 a 50 puntos. Tomando en cuenta los dos grupos, se obtuvo una media de 37.4 y una desviación estándar de 7.44, donde la calificación mínima observada fue de 16 y la máxima de 49, con un coeficiente de asimetría de -0.792 y una curtosis de 0.0934 .

Al analizar por separado al grupo control y al grupo experimental se observó que el grupo experimental obtuvo un mayor puntaje $(M=41.2)$ que el de control $(M=34.4)$. Además, el mismo grupo experimental obtuvo una menor variabilidad $(D E=4.61)$ que el grupo control $(D E=7.93)$. Con respecto a la normalidad en cada grupo, el grupo control mostró una distribución normal (Kolmogorov-Smirnov $=.110, p=.200$; Shapiro-Wilk $=.969, p=.609)$, pero no así el grupo experimental (Kolmogorov-Smirnov $=.233, p=.006$; Shapiro-Wilk $=.882, p=.020$ ). Para determinar si la diferencia entre las medias era significativa, se utilizó una prueba estadística no paramétrica llamada U de Mann-Whitney. Como resultado de la aplicación de esta prueba, se observó que las medias de los grupos fueron significativamente diferentes $(U=123, p=$ .004 ), donde el grupo experimental obtuvo una media significativamente ma- yor $(M=41.2)$ que el grupo control $(M$ $=34.4$ ). Esto indica que la estrategia de motivación utilizada en la enseñanza sirve para mejorar la calificación en el requisito de monografía de los estudiantes analizados. El tamaño del efecto medido con la d de Cohen fue de -1.03, lo cual se considera un efecto grande.

La evaluación final se realizó con una escala de 0 a 100 puntos. Tomando en cuenta los dos grupos, se obtuvo una media de 74.3 y una desviación estándar de 11.8, donde la calificación mínima fue de 46 y la máxima de 94 , con un coeficiente de asimetría de -0.480 y una curtosis de -0.416 .

Al analizar por separado el grupo experimental y el grupo control, se observó que el grupo experimental obtuvo un mayor puntaje y menor variabilidad $(M=78.7, D E=8.34)$ que el de control $(M=70.9, D E=13.1)$, en la mediana (80 el grupo experimental y 67 el grupo control) y en el valor máximo (94 puntos y 92 puntos para los respectivos grupos). Se puede considerar que las dos subpoblaciones muestran distribuciones normales, el grupo experimental (Kolmogorov-Smirnov $=.176, p=.106$; Shapiro - Wilk $=.951, p=.384)$ y el grupo control (Kolgomorov-Smirnov $=.147, p$ $=.169$; Shapiro-Wilk $=.931, p=.091$ ).

Para determinar si la diferencia de rendimiento final entre los grupos fue significativa se buscó determinar si había homogeneidad de varianzas entre ellos. En este caso, las varianzas de los dos grupos fueron diferentes $(F(1,43)=$ $8.22, p=.006$ ), por lo cual, para determinar si existía diferencia significativa de rendimiento entre ellos, se utilizó la prueba estadística $t$ de Welch cuyo resultado mostró que las medias fueron diferentes $(t(41.3)=-2.42, p=.020)$. El grupo experimental obtuvo una puntuación 
significativamente mayor $(M=78.7)$ que el grupo control $(M=70.9)$. Lo anterior indica que la estrategia de motivación utilizada en la enseñanza sirve para mejorar la nota final de los estudiantes analizados. El tamaño de efecto medida con la $d$ de Cohen es de -.692, lo cual es un efecto medianamente grande.

\section{Discusión}

A pesar de que la muestra utilizada fue pequeña y el trabajo puede replicarse con grupos más numerosos de estudiantes, se ha podido apreciar de forma general la influencia que, en el ambiente escolar, tiene el propiciar emociones positivas para un aprendizaje más significativo de los contenidos. Se puntualiza la idea de que el éxito del proceso de enseñanza-aprendizaje está muy vinculado y puede ser sustancialmente modificado por variables subjetivas mediadas por emociones (Kyriacou, 2009). Además, coinciden los resultados presentados con investigaciones donde el índice de éxito escolar está estrechamente relacionado con la satisfacción emocional del estudiante con su ambiente escolar, entre otros factores (Krumrei-Mancuso et al., 2013).

Los resultados del presente estudio confirman que la estrategia de motivación emocional utilizada en la enseñanza sirve para mejorar la nota final de los estudiantes analizados. Esto se refleja en su promedio académico, compuesto por la suma de los diversos productos de aprendizaje sobre los cuales incide la motivación (Edel Navarro, 2003), y ese promedio comprende las notas obtenidas como indicador de logro académico en los diferentes componentes del aprendizaje (Garbanzo Vargas, 2007).

Independientemente de las dificultades metodológicas de los estudios re- visados, Garbanzo Vargas hace énfasis además en diferentes factores que pueden incidir en el rendimiento de los estudiantes frente a sus desafíos académicos y ubica los factores personales como uno de los más importantes. Dentro de estos, la competencia cognitiva y el bienestar psicológico son dignos de tener en cuenta. La competencia cognitiva tiene que ver con las creencias de las capacidades individuales para lograr éxito en las metas y su construcción depende en gran medida del ambiente emocional y de la percepción e interpretación de los propios estados emocionales y somáticos, y precisamente la propiciación de emociones positivas en el aula favorece indirectamente el fortalecimiento de esta competencia. Por otra parte, el bienestar psicológico se ha encontrado altamente relacionado con el rendimiento académico y viceversa (Elizondo Moreno et al., 2018). Si los resultados y logros en el transcurso de la clase y en las diferentes evaluaciones son positivos, aumenta la autoeficacia, la satisfacción y la felicidad asociadas con el estudio (Garbanzo Vargas, 2007), lo cual corrobora nuestro supuesto de que motivar al estudiante mediante ambientes positivos puede mejorar su rendimiento académico.

Generar ambientes emocionales positivos en el aula ayuda a desarrollar las competencias emocionales de los estudiantes y se ha comprobado cómo ellas están relacionadas con su desarrollo académico y el favorecer un clima positivo en el aula aumenta el interés por el aprendizaje y la mejora de las competencias sociales (López-González y Oriol, 2016). Además del ambiente familiar, el ambiente escolar es vital para el desarrollo y la consolidación de la autoestima de los jóvenes, la cual está mediada por sus percepciones emocionales y es otro 
de los factores personales que influye ampliamente en su rendimiento académico (Gutiérrez-Saldaña et al., 2007).

Los resultados obtenidos apoyan la idea de que la estrategia de motivación utilizada en la enseñanza sirve para mejorar la calificación en el requisito de monografía de los estudiantes analizados. La realización de la monografía, como producto del aprendizaje en la materia Métodos de Investigación, requiere de una cuidadosa y reflexiva investigación. El estudiante debe ser capaz de apropiarse de los contenidos ofrecidos en el aula, elaborar su propia idea de investigación, definir las variables de su estudio, diseñar la estrategia y la metodología a seguir para alcanzar sus objetivos, aplicar los instrumentos e interpretar los resultados, además de redactar un informe final que cumpla con los requisitos léxicos y gramaticales, así como de acotación de referencias de acuerdo al estilo aprobado por la universidad (Dawson, 2016; Gastel y Day, 2016; Hernández Sampieri et al., 2014; Turabian et al., 2018).

Para los estudiantes de primer año de la carrera de Teología, representa un desafío la materia de Métodos de Investigación y la monografía es, de las asignaciones, la que más peso aporta a la nota final y a su promedio académico en la clase (50\%). El desarrollo de procesos sistemáticos, críticos y empíricos que se aplican al objeto o fenómeno de estudio de la investigación requiere de los alumnos la presencia de ciertas habilidades y competencias, así como de la percepción de sus propias capacidades. Esto genera un estrés adicional en el estudiante y la tarea del educador es propiciar un ambiente de positividad en el aula que contrarreste las emociones negativas y genere aprendizajes positivos y emocionantes, además de fomentar el aprendiza- je autorregulado (competencia aprender a aprender) para que el alumnado pueda ser autónomo y eficaz en su aprendizaje (Gargallo et al., 2016), teniendo un aprendizaje profundo basado en su deseo de comprender, entender y dominar lo desconocido (Forés Miravalles y Ligioiz Vázquez, 2009). Crear esos ambientes emocionales en contextos educacionales puede ayudar a transformar el aprendizaje de los estudiantes de ser acumulativo y asimilativo a acomodativo y transformativo, implicando estos últimos tipos de aprendizaje la capacidad de romper con las formas tradicionales de resolver los problemas y progresivamente reestructurar el paquete estándar de posibles escenas y patrones de solución en otro nuevo, lo que lleva a la transformación del propio individuo (Erickson, 2019). Todo lo anterior permite mejorar sustancialmente el rendimiento académico y el proceso de enseñanza-aprendizaje (Elizondo Moreno et al., 2018), ya que este es un proceso emocional cuando se mira desde la perspectiva de la neurociencia (Ibarrola, 2014).

Otros aspectos a tener en cuenta cuando se evalúa un trabajo de investigación es el pensamiento crítico y la capacidad de análisis y reflexión (Allen, 2004; Sánchez Upegui, 2011; Weyers y McMillan, 2013), ya que el estudiante debe revisar múltiples fuentes bibliográficas y escoger las que se adecuen a su línea investigativa, además de presentar un proceso de pensamiento crítico redactado apropiadamente y con la calidad que los estudios universitarios requieren (Bañales Faz, 2010). El pensamiento crítico tan necesario en el proceso investigativo requiere que el estudiante sea capaz de trabajar en sus principales ideas, planificar cuidadosamente como las ha de comunicar de una manera lógica y 
entendible, establecer un marco estructural en el que sus hechos se basen y evidencias que les den sentido y establecer vínculos apropiados entre las ideas para presentar sus conclusiones (Allen, 2004) y todas estas decisiones cognitivas están vinculadas a los componentes afectivos de la personalidad.

Las emociones, entonces, se han relacionado extensamente con la capacidad de toma de decisiones en los individuos, con sus capacidades adaptativas de ofrecer respuestas ante los problemas que se presentan (García Retana, 2012) y con las elecciones aparentemente racionales, ya que emoción y razón son interdependientes. Son vitales además en el proceso de motivación y superación de dificultades, aumentan la capacidad de respuesta y el espectro de ellas ante determinadas situaciones, son imprescindibles en la memoria y la evocación agilizando por tanto el aprendizaje, intervienen en los procesos de razonamiento y son consideradas transcendentales en la enseñanza, recomendándose que se asocien a los contenidos a ser impartidos (Forés Miravalles y Ligioiz Vázquez, 2009).

Las teorías que proponen ensanchar y construir el espectro de emociones positivas en la vida diaria presentan, como punto común, expandir el ámbito cognitivo individual para cultivar los recursos personales. Emociones positivas como el gozo, el interés, la gratitud, el asombro y muchas otras desarrolladas a través del programa de intervención presentado en este estudio, expanden los pensamientos y acciones individuales, lo cual estimula la creatividad, la exploración de nuevas formas de hacer las cosas y la resiliencia, que facilitan el desarrollo de los recursos personales y promueven el crecimiento personal y grupal. Ayuda a los estudiantes a incrementar su ha- bilidad para manejar los estresores diarios, promueve el optimismo y facilita el pensamiento realista y la flexibilidad ante los problemas que se encuentren, siendo más asertivos y desarrollando sus competencias (Seligman et al., 2009). Además, los beneficios de estas emociones generadas en el ambiente escolar comienzan a ser acumulativos y actúan sinérgicamente ampliando la capacidad cognitiva y convirtiéndose en un detonador en espiral de éxitos sucesivos en las diferentes esferas de la vida del estudiante (Armenta et al., 2017; Fredrickson, 1998; Seligman et al., 2009).

Con la investigación se concluyó además que, si un alumno tiene una nota alta en la monografía, también tiene una nota alta final. Lo anterior es posible porque la nota final en la materia es la sumativa de las diferentes actividades académicas que el estudiante realiza durante el curso y la monografía tributa en gran medida a esa nota final. Los estudios revisados en el artículo de Barahona U. (2014) se refieren a estudiantes que obtuvieron puntaciones altas en la prueba de ingreso en la universidad y lograron alcanzar un mayor rendimiento académico e incluso concluyó que el rendimiento académico de los años posteriores estaba asociado a las notas obtenidas en el primer año, asumiendo que notas altas en exámenes intermedios tributan a mejores notas finales, lo cual es consistente con los hallazgos de la presente investigación.

Además, hay que tener en cuenta el valor motivacional que los éxitos intermedios pueden tener en el éxito final de la clase y su relación con las emociones positivas. En una investigación realizada con estudiantes de secundaria básica y en materias de ciencias, se pudo comprobar que el obtener buenas calificaciones en 


\section{EMOCIONES POSITIVAS Y RENDIMIENTO ACADÉMICO}

los exámenes produjo y reforzó la presencia de emociones positivas como alegría y felicidad, las cuales también se asociaron a la motivación que les causó realizar actividades prácticas durante las clases (Dávila Acedo et al., 2016). Lo anterior permite concluir que las emociones positivas generan buenos resultados académicos y estos a su vez se convierten en fuente de refuerzo de esas emociones, generando un ambiente de bienestar subjetivo ideal para el aprendizaje significativo.

Es interesante notar que, aunque no era la intención primaria que el programa de intervención aplicado fuera un programa de educación emocional, los objetivos de las actividades motivadoras apuntaron hacia el desarrollo de competencias emocionales, que son el resultado de la educación emocional (López-González y Oriol, 2016). El tratar de describir las emociones personales a través del autoconocimiento, lograr identificar las emociones de los demás, desarrollar la habilidad para generar emociones positivas y reconocerlas, desarrollar la habilidad de automotivarse y adoptar una actitud positiva ante los desafíos de la vida y del ambiente escolar (Bisquerra Alzina, 2009) son objetivos encaminados a desarrollar competencias emocionales que estuvieron presentes en las actividades del programa de intervención.

Se propone incentivar el uso de las herramientas que la psicología positiva ofrece en función de la educación integral, al reproducir este tipo de intervenciones generadoras de emociones positivas en contextos educativos más amplios. Los dividendos serán mucho más abarcantes que un mejor rendimiento académico, pues el aumento del bienestar subjetivo con todos sus beneficios se incrementa proporcionalmente con este tipo de actividades (Fredrickson, 1998; Lyubomirsky et al., 2011; Seligman et al., 2009).

\section{Referencias}

Adelman, J. S. y Estes, Z. (2013). Emotion and memory: A recognition advantage for positive and negative words independent of arousal. Cognition, 129(3), 530-535. https://doi.org/10.1016/j. cognition.2013.08.014

Allen, M. (2004). Smart thinking: Skills for critical understanding and writing (2a. ed.). Oxford University Press.

Armenta, C. N., Fritz, M. M. y Lyubomirsky, S. (2017). Functions of positive emotions: Gratitude as a motivator of self-improvement and positive change. Emotion Review, 9(3), 183-190. https://doi. org/10.1177/1754073916669596

Bañales Faz, G. (2010). Escritura académica en la universidad: regulación del proceso de composición, conocimientos del tema y calidad textual [Tesis doctoral, Universidad Ramón Liull]. Tesis doctorals en Xarxa. https://www.tdx.cat/10803/9281

Barahona U., P. (2014). Factores determinantes del rendimiento académico de los estudiantes de la Universidad de Atacama. Estudios Pedagógicos, 40(1), 25-39. https://doi.org/10.4067/S071807052014000100002

Bender, Y. (2004). The power of positive teaching: 35 successful strategies for active and enthusiastic classroom participation. Nomad Press.

Bisquerra Alzina, R. (2009). Psicopedagogía de las emociones. Síntesis. https://www.sintesis.com

Cohn, M. A., Fredrickson, B. L., Brown, S. L., Mikels, J. A. y Conway, A. M. (2009). Happiness unpacked: Positive emotions increase life satisfaction by building resilience. Emotion, 9(3), 361-368. https://doi. org/10.1037/a0015952

Csikszentmihalyi, M. (2014). Flow and the foundations of positive psychology: The collected works of Mihaly Csikszentmihalyi. Springer Netherlands. https://doi.org/10.1007/978-94-017-9088-8

Dávila Acedo, M. A., Cañada Cañada, F., Sánchez Martín, J. y Mellado Jiménez, V. (2016). Las emociones en el aprendizaje de física y química en educación secundaria: Causas relacionadas con el estudiante. Educación Química, 27(3), 217-225. https://doi.org/10.1016/j.eq.2016.04.001 


\section{VIVES IGLESIAS ET AL.}

Dawson, C. (2016). 100 activities for teaching research methods. Sage. https://study.sagepub.com/ catherinedawson

Edel Navarro, R. (2003). El rendimiento académico: Concepto, investigación y desarrollo. Revista Electrónica Iberoamericana sobre Calidad, Eficacia y Cambio en Educación, 1(2). http://www.ice. deusto.es/rinace/reice/vol1n2/Edel.pdf

Elizondo Moreno, A., Rodríguez Rodríguez, J. V. y Rodríguez Rodríguez, I. (2018). La importancia de la emoción en el aprendizaje: Propuestas para mejorar la motivación de los estudiantes. Cuaderno de Pedagogía Universitaria, 15(29), 3-11. https://doi.org/10.29197/cpu.v15i29.296

Erickson, A. R. (2019). Human learning, memory, and student development. Contemporary Issues in Educational Leadership, 8, 1-15. https://doi.org/10.32873/unl.dc.ciel.1010

Fernández-Lasarte, O., Ramos-Díaz, E. y Axpe Sáez, I. (2019). Rendimiento académico, apoyo social percibido e inteligencia emocional en la universidad. European Journal of Investigation in Health, Psychology and Education, 9(1), 39-49. https://doi.org/10.30552/ejihpe.v9i1.315

Forés Miravalles, A. y Ligioiz Vázquez, M. (2009). Descubrir la neurodidáctica: aprender desde, en y para la vida. UOC.

Franco Cedeño, F. J., Osorio Sánchez, A. V. y Cervantes Molina, X. P. (2019). Relación entre el bienestar psicológico, rendimiento académico y acoso en los estudiantes universitarios. Universidad y Sociedad, 11(5),301-308. http://scielo.sld.cu/scielo.php?script=sci_arttext\&pid=S2218-36202019000500301\&ln $\mathrm{g}=\mathrm{es} \& \mathrm{nrm}=\mathrm{iso} \& \mathrm{tlng}=\mathrm{es}$

Frazzetto, G. (2014). Cómo sentimos: sobre lo que la neurociencia puede y no puede decirnos acerca de nuestras emociones. Anagrama.

Fredrickson, B. L. (1998). What good are positive emotions? Review of General Psychology, 2(3), 300319. https://doi.org/10.1037/1089-2680.2.3.300

Fredrickson, B. L. (2016). Positive emotions broaden and build. En L. F. Barrett, M. Lewis y J. M. HavilandJones (Eds.), Handbook of emotions (Vol. 47, pp. 1-53). Guilford Press. https://doi.org/10.1016/B9780-12-407236-7.00001-2

Fredrickson, B. L., Cohn, M. A., Coffey, K. A., Pek, J. y Finkel, S. M. (2008). Open hearts build lives: Positive emotions, induced through loving-kindness meditation, build consequential personal resources. Journal of Personality and Social Psychology, 95(5), 1045-1062. https://doi.org/10.1037/ a0013262

Fredrickson, B. L. y Joiner, T. (2018). Reflections on positive emotions and upward spirals. Perspectives on Psychological Science, 13(2), 194-199. https://doi.org/10.1177/1745691617692106

Fredrickson, B. L., y Losada, M. F. (2005). Positive affect and the complex dynamics of human flourishing. The American Psychologist, 60(7), 678-686. https://doi.org/10.1037/0003-066X.60.7.678

Garbanzo Vargas, G. M. (2007). Factores asociados al rendimiento académico en estudiantes universitarios: Una reflexión desde la calidad de la educación superior pública. Revista Educación, 31(1), 43-63. https://doi.org/10.15517/REVEDU.V31I1.1252

García Retana, J. A. (2012). La educación emocional, su importancia en el proceso de aprendizaje. Revista Educación, 36(1), 97-109. https://doi.org/10.15517/REVEDU.V36I1.455

Gargallo, B., Campos, C. y Almerich, G. (2016). Learning to learn at university: The effects of an instrumental subject on learning strategies and academic achievement. Cultura y Educación, 28(4), 771-810. https://doi.org/10.1080/11356405.2016.1230293

Gastel, B. y Day, R. A. (2016). How to write and publish a scientific paper. Greenwood.

Gómez Chacón, I. M. (2000). Matemática emocional: los afectos en el aprendizaje matemático. Narcea.

Gutiérrez-Saldaña, P., Camacho-Calderón, N. y Martínez-Martínez, M. L. (2007). Autoestima, funcionalidad familiar y rendimiento escolar en adolescentes. Atención Primaria, 39(11), 597-601. https://doi.org/10.1157/13112196

Hernández Sampieri, R., Fernández Collado, C. y Baptista Lucio, M. P. (2014). Metodología de la investigación (6a. ed.). McGraw-Hill.

Hill, N. y Stone, W. C. (1982). Success through a positive mental attitude (2a. ed.). Debolsillo.

Hooper, J. (2012). What children need to be happy, confident and successful: Step by step positive psychology to help children flourish. Jessica Kingsley Publishers.

Ibarrola, B. (2014). Aprendizaje emocionante: Neurociencia para el aula. Innovación educativa. SM.

Immordino-Yang, M. H., McColl, A., Damasio, H. y Damasio, A. R (2009). Neural correlates of admiration and compassion. Proceedings of the National Academy of Sciences, 106(19), 8021-8026. https://doi. org/10.1073/pnas.0810363106 


\section{EMOCIONES POSITIVAS Y RENDIMIENTO ACADÉMICO}

Krumrei-Mancuso, E. J., Newton, F. B., Kim, E. y Wilcox, D. (2013). Psychosocial factors predicting firstyear college student success. Journal of College Student Development, 54(3), 247-266. https://doi. org $/ 10.1353 / \mathrm{csd} .2013 .0034$

Kyriacou, C. (2009). Effective teaching in schools: Theory and practice (3a. ed.). Nelson Thornes.

López-González, L. y Oriol, X. (2016). The relationship between emotional competence, classroom climate and school achievement in high school students. Cultura y Educación, 28(1), 130-156. https://doi.org/10.1080 $/ 11356405.2015 .1120448$

Lyubomirsky, S., Dickerhoof, R., Boehm, J. K. y Sheldon, K. M. (2011). Becoming happier takes both a will and a proper way: An experimental longitudinal intervention to boost well-being. Emotion, 11(2), 391-402. https://doi.org/10.1037/a0022575

MacConville, R. y Rae, T. (2012). Building happiness, resilience and motivation in adolescents: A positive psychology curriculum for well-being. Jessica Kingsley Publishers.

Ormrod, J. E. (2005). Aprendizaje humano (4a. ed.). Pearson-Prentice Hall.

Ortiz Alonso, T. (2018). Neurociencia en la escuela: Hervat: investigación neuroeducativa para la mejora del aprendizaje. SM.

Rolla, A., Hinton, C. y Shonkoff, J. (2011). The science of early childhood development. Hacia un modelo interdisciplinario: biología, interacción social y desarrollo infantil temprano. Jorge Baudino Ediciones.

Rusk, R. D. y Waters, L. E. (2013). Tracing the size, reach, impact, and breadth of positive psychology. The Journal of Positive Psychology, 8(3), 207-221. https://doi.org/10.1080/17439760.2013.777766

Saklofske, D. H., Austin, E. J., Mastoras, S. M., Beaton, L. y Osborne, S. E. (2012). Relationships of personality, affect, emotional intelligence and coping with student stress and academic success: Different patterns of association for stress and success. Learning and Individual Differences, 22(2), 251-257. https://doi.org/10.1016/j.lindif.2011.02.010

Sánchez-López, D., León-Hernández, S. R. y Barragán-Velásquez, C. (2015). Correlación de inteligencia emocional con bienestar psicológico y rendimiento académico en alumnos de licenciatura. Investigación en Educación Médica, 4(15), 126-132. https://doi.org/10.1016/j.riem.2015.04.002

Sánchez Upegui, A. A. (2011). Manual de redacción académica e investigativa: cómo escribir, evaluar y publicar artículos. Católica del Norte Fundación Universitaria.

Seligman, M. E. P. (2011). Flourish: A visionary new understanding of happiness and well-being. William Heinemann.

Seligman, M. E. P., Ernst, R. M., Gillham, J., Reivich, K. y Linkins, M. (2009). Positive education: Positive psychology and classroom interventions. Oxford Review of Education, 35(3), 293-311. https://doi. org $/ 10.1080 / 03054980902934563$

Sheldon, K. M. y Lyubomirsky, S. (2006). Achieving sustainable gains in happiness: Change your actions, not your circumstances. Journal of Happiness Studies, 7(1), 55-86. https://doi.org/10.1007/s10902-005-0868-8

Shindler, J. (2010). Transformative classroom management: Positive strategies to engage all students and promote a psychology of success. Jossey-Bass.

Solano Luengo, L. O. (2015). Rendimiento académico de los estudiantes de Secundaria Obligatoria y su relación con las aptitudes mentales y las actitudes ante el estudio [Tesis doctoral, UNED]. e-spacio. http://e-spacio.uned.es/fez/view/tesisuned:Educacion-Losolano

Tamblyn, D. (2003). Laugh and learn: 95 ways to use humor for more effective teaching and training. Amacom.

Tarragona, M. (2013). Psicología positiva y terapias constructivas: Una propuesta integradora. Terapia Psicológica, 31(1), 115-125. https://doi.org/10.4067/S0718-48082013000100011

Tarragona, M. (2014). Tu mejor tú: Cómo la psicología positiva te enseña a subrayar las experiencias que fortalecen tu identidad. Alianza Editorial.

Turabian, K. L., Booth, W. C., Colomb, G. G., Williams, J. M., Bizup, J. y FitzGerald, W. T. (2018). A manual for writers of research papers, theses, and dissertations: Chicago style for students and researchers (9a. ed.). The University of Chicago Press.

Walsh, L. C., Boehm, J. K. y Lyubomirsky, S. (2018). Does happiness promote career success? Revisiting the evidence. Journal of Career Assessment, 26(2), 199-219. https://doi.org/10.1177/1069072717751441

Westwood, P. S. (2008). Teaching methods: What teachers need to know about. ACER Press.

Weyers, J. y McMillan, K. (2013). How to improve your critical thinking and reflective skills. Pearson.

Recibido: 4 de agosto de 2020

Revisado: 2 de diciembre de 2020

Aceptado: 9 de diciembre de 2020 